\title{
Superlative-modified numerals and negation: A multiply negotiable cost
}

\author{
Teodora Mihoc \& Kathryn Davidson*
}

\begin{abstract}
Comparative-modified numerals (CMNs) and superlative-modified numerals (SMNs) are reported to exhibit a polarity sensitivity contrast: unlike CMNs, the use of SMNs is said to be sensitive to embedding under negation. This contrast is however neither well studied nor well understood, such that the existing views in the literature disagree vastly on both the basic facts and related expectations. In this paper we investigate this contrast in three offline experiments. We show that there is strong empirical support for this reported contrast under negation, but none of the existing analyses of the contrast can capture it in full, though we do seem to require insights from each.
\end{abstract}

Keywords. comparative-modified numerals, superlative-modified numerals; negation

\section{Introduction.}

1.1. THE POLARITY SENSITIVITY CONTRAST. Naively speaking, given reference to the same scale, comparative-modified numerals (CMNs; e.g., more/less than 3) and superlative-modified numerals (SMNs; e.g., at least/most 3) are pairwise truth-conditionally equivalent (more than $2=$ at least $3=3$ or 4 or ... ). However, they are reported to differ in multiple ways. One reported contrast has to do with polarity sensitivity. This contrast is usually described as follows: CMNs are fine in all downward-entailing (DE) environments, but SMNs have a mixed profile: their acceptability is degraded in some DE environments, for example a negative declarative (DECL-NEG), but acceptable in other DE environments, for example, the positive antecedent of a conditional (ANTCOND-POS) or restriction of a universal (RESTUniv-Pos) (Geurts \& Nouwen 2007, a.o.).

(1) Jo didn't call $\checkmark$ more than $2 /$ \# at least 3 people.

$($ not $>\sqrt{ } \mathrm{CMN} / \# \mathrm{SMN})$

(2) If Jo called $\sqrt{ }$ more than $2 / \sqrt{ }$ at least 3 people, she passed.

$($ if $>\sqrt{ } \mathrm{CMN} / \sqrt{\mathrm{SMN}})$

(3) Everyone who called $\sqrt{ }$ more than $2 / \sqrt{ }$ at least 3 people passed. (every $>\sqrt{ } \mathrm{CMN} / \sqrt{ } \mathrm{SMN}$ )

1.2. Four theORETICAL POSITIONS. The existing literature implicitly or explicitly espouses the following four views with respect to the polarity sensitivity contrast. Except for the first one, which denies the contrast, each of these views has a story for the contrast in DECL-NEG and the non-contrast in ANTCOND/RESTUNIV-POS, as well as predictions for a negative antecedent of a conditional / restriction of a universal (ANTCOND/RESTUNIV-NEG: If Jo didn't call at least 3 people, she passed / Everyone who didn't call at least 3 people passed) and at least one other prediction. We briefly review each below.

V0: No CONTRAST. This (admittedly straw-man) view holds that there is no contrast.

V1: Processing COST. This view is as follows: SMNs are degraded in DECL-NEG and fine in AntCond/RESTUniv-Pos because SMNs incur an extra processing cost (see, e.g., Alexan-

*We would like to thank Athulya Aravind, Gennaro Chierchia, Andreea Nicolae, and Steven Worthington (The Institute of Quantitative Social Sciences at Harvard), as well as members of the Experimental Syntax \& Semantics Lab / Language Acquisition Lab at MIT and of the Meaning and Modality Lab and/or of the Language and Cognition Lab at Harvard, and audiences at XPrag 2017 and ELM 1. Any errors are our own. Authors: Teodora Mihoc, Harvard University (tmihoc@fas.harvard.edu) \& Kathryn Davidson, Harvard University (kathryndavidson@fas.harvard.edu). 
dropoulou 2018 and refs. therein) and the first environment contains negation, which adds to this cost (Wason 1961, Clark \& Chase 1972), but the other two environments do not. This view predicts that SMNs in ANTCOND/RESTUNIV-NEG should be degraded also, perhaps even more, as these environments not only contain negation but are also even more complex. This view also suggests that the DE modifiers might also generally be worse, as they are also in some sense negative.

V2: Monotonicity And Evaluativity (COHEN \& KRIFKA 2014). This view goes as follows: SMNs are degraded in DECL-NEG and fine in ANTCOND/RESTUNIV-Pos because they have two lexical meanings, one sensitive to monotonicity, which requires that the SMN be in an upward-entailing (UE) environment, a condition violated in both DECLNEG and ANTCOND/RESTUNIV-Pos above, and one sensitive to evaluativity, which requires that the property that the SMN combines with be pragmatically positive, a condition met in ANTCOND/RESTUNIV-POS above, where the positive continuation passed forces the otherwise neutral call people to be understood as positive. This view predicts that, on the meaning sensitive to monotonicity, SMNs in ANTCOND/RESTUNIV-NEG should always be fine, as these doubly DE environments are altogether UE. This view also predicts that, on the meaning sensitive to evaluativity, an SMN in either ANTCOND/RESTUNIV-POS or ANTCOND/RESTUNIV-NEG may be degraded: If you click at least twice, \# the system will crash / If you don't click at least twice, \# you will get a discount is degraded because the negative / positive continuation forces the antecedent to be understood as negative / positive, which forces the predicate click to be understood as negative.

V3: Monotonicity Alone (Spector 2015, Minoc 2020). This view goes as follows: SMNs are degraded in DECL-NEG and fine in ANTCOND/RESTUNIV-POS because SMNs are sensitive to downward monotonicity and the first environment is DE at all levels whereas the other two actually contain an UE presupposition (e.g., If Jo called... / Everyone who called. . . presuppose that it is possible that Jo/someone called). This view predicts that SMNs should be fine in AntCond/RESTUniv-NEG, as these environments are UE. This view also predicts that SMNs should be fine, for example, under a negated factive (e.g., Tim doesn't know that Jo called ... ), as this also yields a DE environment with an UE presupposition (that Jo called ... ), or under two negations (e.g., Tim doesn't know that Jo didn't call ... ), as this also yields an UE environment.

1.3. GOAL OF THIS PAPER AND PLAN. The four views above are very different. In this paper we report on three offline experiments in which we tested their basic assumptions and expectations. In Exp. 1 we checked the basic patterns, trying to decide between V0 and V1-3 and between V1 and V2-3 (§2). In Exp. 2, we tested a prediction from V2 (\$3). And in Exp. 3 we tested a prediction from V3 (§4). As we show, our findings reject V0 and support a mixture of V1-3 (§5).

\subsection{General NOTES ON METhODS AND RESUlts.}

MEthods. The sentences we wanted to test pose a number of specific challenges: (1) These sentences are complex, with multiple logical operators, and inherently awkward. (2) At least in a positive declarative, the epistemic state of the speaker might introduce a known confound (SMNs require an ignorant speaker, though $\mathrm{CMNs}$ are fine with either knowledge or ignorance; Nouwen et al. 2019 and refs. therein). (3) In a negative declarative the target narrow scope, how many?, reading can be avoided by interpreting the SMN with a wide scope, specific reading (Mayr 2013). To address all these points, we decided to adopt a card game scenario inspired by Cremers \& Chemla (2017), making it clear that the characters in the game are always talking about how many?

Teodora Mihoc and Kathryn Davidson:

Superlative-modified numerals and negation: A multiply negotiable cost. 
cards, and never about specific cards, and keeping the epistemic state of the speaker transparent and constant. Aside from these, an additional challenge is as follows: (4) The nature of the polarity sensitivity contrast is not clear-these sentences appear to be syntactically well-formed and, with some effort, one can compute their meaning. To address this, we decided to ask participants to provide what we term comprehensibility judgments: Do you think $x$ will understand what y said? All of (1)-(4) are enforced via pictures in every trial. In addition to these, there were further difficulties, namely: (5) Varying the numeral might add further complexity. To address this we decided to keep it constant (always three). Finally, (6) The contrast is fairly subtle. To maximize chances of detecting it, since we had no reason to be concerned about conscious access to the task affecting responses, we chose not to use any fillers, and merely to present the stimuli in a new, random order each time. Concrete examples of the tasks and stimuli are given in what follows, and printouts of the full surveys online at https://osf.io/6gpu3/. Finally: (7) We wanted a diverse population. To address this, we recruited participants from Amazon Mechanical Turk (self-reported native speakers of English, different for each of Exp. 1-3, and paid \$2, \$1, and \$1), and provided a link to the survey, presented via Qualtrics software.

RESULTS. The results were analyzed in R. In each case we first report the raw results in the form of a plot, then also the result of fitting logistic mixed effects regression models, commenting in particular on general effects, contrasts between the two modifier types, and contrasts within the modifier types. Our data and analysis scripts are available at https://osf . io/6gpu $3 /$.

\section{Experiment 1.}

2.1. Goal. To test the basic patterns for DeCL-Neg and AntCond/RESTUniv-Pos, as well as for ANTCOND/RESTUNIV-NEG, as a way to tease apart V0 from V1-3 and V1 from V2-3.

2.2. Methods. Task: See Figure 1a. Sample trial: See Figure 1b. Trial summary: There were 24 trials, obtained by crossing the following factors: Env = embedding environment (DECL = declarative; ANTCOND = antecedent of conditional; RESTUNIV = restriction of universal); Pol $=$ polarity of Env $(\mathrm{POS}=$ positive, $\mathrm{NEG}=$ negative $)$; and ModType $=$ modifier type $(\mathrm{COMP}=$ comparative, SUP = superlative $) \times$ ModMon = modifier monotonicity $(\mathrm{UE}, \mathrm{DE})$, which yield Mod = modifier (MoreThan, Lessthan, AtLeast, AtMost). See Figure 1c. Participants: 99, of which 3 were excluded (due to unrealistic end time, or providing the same answer in all trials).

2.3. RESUlts. For the raw means see Figure 1d and for model results see Tables 1a-1c. General effects: For all environment types, and for both CMNs and SMNs, ratings were significantly lower for ModMon=DE, Pol=NEG, the interaction of ModMon=DE with Pol=NEG, and also (marginally) for Env=REstUniv. See Table 1a. Contrasts between Modifier Types: For the same level of monotonicity, SMNs in DECL-POs were the same as CMNs; in DECL-NEG were much worse than CMNs; in a ANTCOND/RESTUNIV-POS were the same as CMNs, except for $a t$ most in a ReSTUniv-Pos, which was worse; and in AnTCONT/RESTUniv-NeG were slightly worse than CMNs. See Table 1b. Contrasts within Modifier Types: CMNs degraded from DECLNeg to AntCond/RestUniv-Neg, but SMNs did not. See Table 1c.

2.4. DisCUSSION. SMNs were much worse than CMNs in DECL-NEG but on a par with CMNs in ANTCOND/RESTUNIV-POS. This argues strongly against V0 and in favor of V1-3. SMNs were in fact significantly worse than CMNs in every NEG condition. This might seem to support V1

Teodora Mihoc and Kathryn Davidson:

Superlative-modified numerals and negation: A multiply negotiable cost. 
(a) Exp. 1 task.

In this survey you will answer questions about a group of friends playing a game. At the beginning of the game each player gets dealt a hand of seven cards. After taking a quick look at them, they must place the cards face down and try to remember their hands. Then they take turns giving clues about their hands to the other players in the form of statements describing their hands. You will see what a player remembers about his/her cards and the statement s/he makes, then you will be asked if you think the other players will understand what s/he said.

Note: a or a means that the player doesn't remember if a particular card in his hand was a club or a spade, or a diamond or a heart, respectively.

(b) Exp. 1 sample trial. Answer options: Yes/No. The epistemic state was as illustrated across all trials.

Charizard remembers:

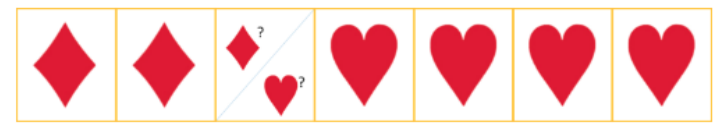

Charizard says: I don't have at most 3 hearts.

Do you think the other players will understand what he said?

(c) Exp. 1 trial summary. All participants saw all trials, in random order.

\begin{tabular}{lll}
\hline Env & Pol & ModType (COMP, SUP) x ModMon (UE, DE) = Mod \\
\hline \multirow{2}{*}{ DECL } & POS & I have Mod 3 [suit]. \\
\cline { 2 - 3 } & NEG & I don't have Mod 3 [suit] \\
\hline \multirow{2}{*}{ ANTCOND } & POS & If you have Mod 3 [suit], then we have something in common. \\
\cline { 2 - 3 } & NEG & If you don't have Mod 3 [suit], then we have something in common. \\
\hline \multirow{2}{*}{ RESTUNIV } & POS & Everyone who has Mod 3 [suit] has something in common with me. \\
\cline { 2 - 3 } & NEG & Everyone who doesn't have Mod 3 [suit] has something in common with me. \\
\hline
\end{tabular}

(d) Exp. 1 raw means and their associated 95\% binomial CIs. $\mathrm{n}=96$.
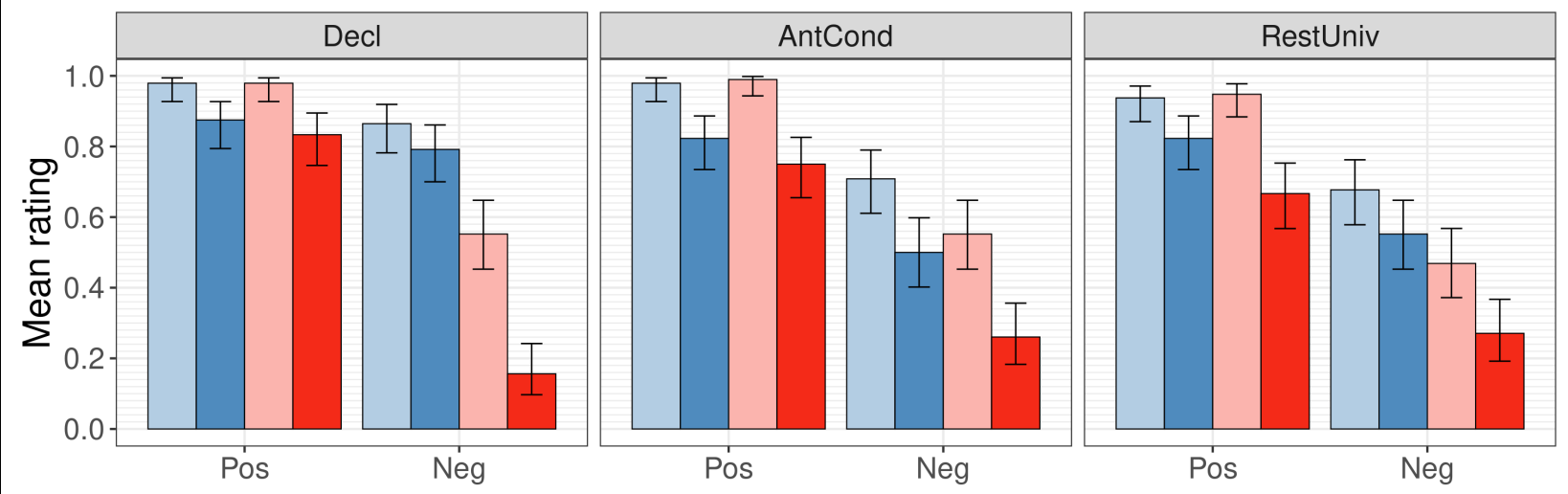

MoreThan

LessThan

AtLeast

AtMost

Figure 1: Exp. 1 (a) instructions, (b) sample trial, (c) trial summary, and (d) raw results.

Teodora Mihoc and Kathryn Davidson:

Superlative-modified numerals and negation: A multiply negotiable cost. 
(a) Exp. 1 predicted effects - general (abridged, but listing all the significant contrasts).

\begin{tabular}{rrrrr}
\hline & Estimate & Std. Error & $\mathrm{z}$ value & $\operatorname{Pr}(>|\mathrm{z}|)$ \\
\hline (Intercept) & 6.30 & 0.93 & 6.795 & $<0.0001$ \\
ModMonDE & -3.58 & 0.90 & -3.978 & 0.0001 \\
ModTypeSup & -0.59 & 1.11 & -0.532 & 0.5949 \\
PolNeg & -3.77 & 0.92 & -4.103 & $<0.0001$ \\
EnvRestUniv & -1.68 & 0.95 & -1.759 & 0.0785 \\
ModMonDE:PolNeg & 2.67 & 0.97 & 2.746 & 0.0060 \\
ModTypeSup:PolNeg & -1.69 & 1.17 & -1.442 & 0.1492 \\
\hline
\end{tabular}

(b) Exp. 1 predicted effects - between Modifier Types (separated by Modifier Monotonicity).

\begin{tabular}{llcrcrr}
\hline Env & Pol & Mod & OR & CI & $\mathrm{z}$ & $\mathrm{p}$ \\
\hline Decl & Pos & MoreThan-AtLeast & 1.80 & {$[0.13,25.63]$} & 0.532 & 1.0000 \\
Decl & Pos & LessThan-AtMost & 2.02 & {$[0.61,6.64]$} & 1.413 & 0.1576 \\
Decl & Neg & MoreThan-AtLeast & 9.73 & {$[3.29,28.83]$} & 5.016 & $<0.0001$ \\
Decl & Neg & LessThan-AtMost & 40.68 & {$[12.66,130.78]$} & 7.597 & $<0.0001$ \\
AntCond & Pos & MoreThan-AtLeast & 0.84 & {$[0.04,19.27]$} & -0.135 & 1.0000 \\
AntCond & Pos & LessThan-AtMost & 2.13 & {$[0.76,5.96]$} & 1.768 & 0.1540 \\
AntCond & Neg & MoreThan-AtLeast & 2.86 & {$[1.13,7.19]$} & 2.722 & 0.0065 \\
AntCond & Neg & LessThan-AtMost & 4.56 & {$[1.77,11.72]$} & 3.842 & 0.0001 \\
RestUniv & Pos & MoreThan-AtLeast & 1.42 & {$[0.24,8.37]$} & 0.477 & 1.0000 \\
RestUniv & Pos & LessThan-AtMost & 3.56 & {$[1.32,9.62]$} & 3.054 & 0.0068 \\
RestUniv & Neg & MoreThan-AtLeast & 3.59 & {$[1.45,8.88]$} & 3.385 & 0.0014 \\
RestUniv & Neg & LessThan-AtMost & 5.32 & {$[2.10,13.53]$} & 4.294 & $<0.0001$ \\
\hline
\end{tabular}

(c) Exp. 1 predicted effects - within Modifier Types (separated by Modifier Monotonicity).

\begin{tabular}{llcrcrl}
\hline Env & Pol & Mod & OR & CI & $\mathrm{z}$ & $\mathrm{p}$ \\
\hline Decl-AntCond & Neg & MoreThan & 3.37 & {$[1.09,10.42]$} & 2.577 & 0.0199 \\
Decl-RestUniv & Neg & MoreThan & 4.19 & {$[1.38,12.73]$} & 3.092 & 0.0060 \\
Decl-AntCond & Neg & LessThan & 5.07 & {$[1.94,13.25]$} & 4.045 & 0.0002 \\
Decl-RestUniv & Neg & LessThan & 3.78 & {$[1.47,9.74]$} & 3.371 & 0.0015 \\
Decl-AntCond & Neg & AtLeast & 0.99 & {$[0.43,2.29]$} & -0.030 & 0.9760 \\
Decl-RestUniv & Neg & AtLeast & 1.55 & {$[0.68,3.52]$} & 1.272 & 0.5521 \\
Decl-AntCond & Neg & AtMost & 0.57 & {$[0.19,1.67]$} & -1.253 & 0.4202 \\
Decl-RestUniv & Neg & AtMost & 0.50 & {$[0.17,1.43]$} & -1.587 & 0.3373 \\
\hline
\end{tabular}

Table 1: Exp. 1 model results. $y \sim$ ModMon * ModType * Pol * Env $+(1+($ ModMon + ModType + Pol + Env) $\mid$ Participant). (Statistically significant results in gray.)

over V2-3. However, this contrast was much larger in DECL-NEG than in ANTCOND/RESTUNIVNEG, due to the fact that, while CMNs degrade between these conditions, SMNs do not. This is inexplicable on V1, where SMNs in ANTCOND/RESTUNIV-NEG are expected to be even worse, just like CMNs, but consistent with V2-3, where SMNs are actually expected to improve. We also failed to find a general penalty for SMNs. Altogether, this suggests that the specific interaction of SMNs with negation is explained not by V1 but rather by V2-3. However, we also found a general penalty for the modifier monotonicity being DE and for negation, and for their interaction. This suggests that V1 always plays a general role too. The fact that we also found a general trend for a penalty for the environment being the restriction of a universal is surprising on all of V1-3.

Teodora Mihoc and Kathryn Davidson:

Superlative-modified numerals and negation: A multiply negotiable cost. 


\section{Experiment 2.}

3.1. GoAL. To check what happens to SMNs in AnTCOND/RESTUniV-Pos/NEG if we vary the pragmatic polarity of the predicate in the continuation, as a way to test the basic insight behind V2 that the key to SMNs in ANTCOND/RESTUNIV lies with questions of evaluativity. If V2 is correct, we expect this manipulation to make a big difference. In particular, an SMN with a pragmatically neutral predicate in ANTCOND/RESTUNIV-POS should be fine with a positive continuation, and in ANTCOND/RESTUNIV-NEG should be fine with a negative continuation.

3.2. Methods. Task: Similar to Exp. 1, adapted to support minimal manipulations of pragmatic predicate polarity in the continuation. See Figure 2a. Sample trial: See Figure 2b. Trial summary: Each participant saw 32 trials, obtained by crossing the following factors: Env = embedding environment (ANTCOND = antecedent of conditional; RESTUNIV = restriction of universal); Pol1 $=$ polarity of Env $(\mathrm{POS}=$ positive, $\mathrm{NEG}=$ negative $) ; \mathrm{Pol} 2=$ pragmatic polarity of predicate in the continuation $(\mathrm{POS}=$ positive; $\mathrm{NEG}=$ negative $)$; and ModType $\mathrm{x}$ ModMon $=\operatorname{Mod}($ as before $)$. See Figure 2c. Participants: 45, of which 5 excluded (same answer in all trials).

3.3. Results. For the raw means see Figure $2 \mathrm{~d}$ and for model results see Tables $2 \mathrm{a}-2 \mathrm{c}$. General effects: For both environment types, and for both CMNs and SMNs, ratings were significantly lower for Pol1=NEG, the interaction of ModMon=DE, ModType=SuP, and Pol2=NEG, the interaction of all the previous factors with Env=RESTUNIV, and the interaction of all the previous factors with Pol1=Neg. See Table 2a. Contrasts between Modifier Types: Compared to its CMN counterpart more than, the UE SMN at least: in Pos-Pos was similar; in Pos-NEG was similar in ANTCOND but worse in RESTUNIV; in NEG-POS was worse; and in NEG-NEG was similar. Compared to its CMN counterpart less than, the DE SMN at most was worse in every condition, although across the two environment types the magnitude of the contrast varied as follows: Pos-Neg $>$ Pos-Pos $>$ Neg-Pos $>$ NEG-Neg. See Table 2b. Contrasts within Modifier Types: Ratings were high for all the modifiers in POS-POS (with the notable exception of at most in RESTUNIV), and generally remained so in POS-NEG also-except for at most, which degraded dramatically (including in the case of RESTUNIV). And ratings were lower for all the modifiers in NEG-POS, and generally remained so in NEG-NEG also-except for at least, which improved dramatically. See Table 2c.

3.4. Discussion. Ratings for SMNs in AntCond/REstUniv-Pos/NeG were affected by the pragmatic polarity of the continuation, both at least and at most being sometimes a lot better or a lot worse as a result of it. This is completely surprising on V1 or V3 but precisely as expected on V2. However, the patterns for at least and at most diverged vastly, and neither followed exactly the expectations from V2. This suggests that SMNs in ANTCOND/RESTUNIV-POS/NEG are indeed sensitive to notions of evaluativity, as expected on V2, but the monotonicity of the modifier also makes a (big) difference. We also found a general penalty for negation, which again supports a general role of V1, and also a small penalty for the environment being the restriction of a universal (possibly specific to SMN though the trend is not clear), which is again surprising on all of V1-3. We did not find a general penalty for the modifier being DE, though this is likely still there, being simply obscured by the strong interaction of SMN modifier monotonicity with the two kinds of polarity.

Teodora Mihoc and Kathryn Davidson:

Superlative-modified numerals and negation: A multiply negotiable cost. 
(a) Exp. 2 task.

In this survey you will answer questions about a group of friends playing a game. At the beginning of the game each player gets dealt a hand of seven cards. They are not allowed to see their own cards but they are allowed to take a quick look at their neighbor's hand. They try to remember their neighbor's hand as well as they can because in the next step they have to come up with a rule that would make that neighbor (and possibly other players too) lose or win. You will see what a player remembers about their neighbor's hand and the rule they make up, then you will be asked if you think the other players will understand what they said. Note, we're not asking you if it is a good rule or a bad rule, but whether it is a rule that is going to be understandable for the other players to follow.

Note: a or a means that the player doesn't remember if a particular card in his hand was a club or a spade, or a diamond or a heart, respectively.

(b) Exp. 2 sample trial. Answer options: Yes/No. The epistemic state was as illustrated across all trials.

Meowth remembers:

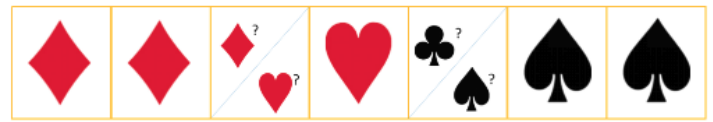

Meowth says: If you don't have at least 3 hearts, you lose.

Do you think the other players will understand what he said?

(c) Exp. 2 trial summary. All participants saw all trials, in random order.

\begin{tabular}{llll}
\hline Env & Pol1 & Pol2 & ModType (COMP, SUP) x ModMon (UE, DE) = Mod \\
\hline \multirow{2}{*}{ ANTCOND } & Pos & Pos/NEG & If you have Mod 3 [suit], you win/lose. \\
\cline { 2 - 5 } & Neg & Pos/NEG & If you don't have Mod 3 [suit], you win/lose. \\
\hline \multirow{2}{*}{ RestUniv } & Pos & Pos/NEG & Everyone who has Mod 3 [suit] wins/loses. \\
\cline { 2 - 4 } & NEG & Pos/NEG & Everyone who doesn't have Mod 3 [suit] wins/loses. \\
\hline
\end{tabular}

(d) Exp. 2 raw means and their associated 95\% binomial CIs. $\mathrm{n}=40$.
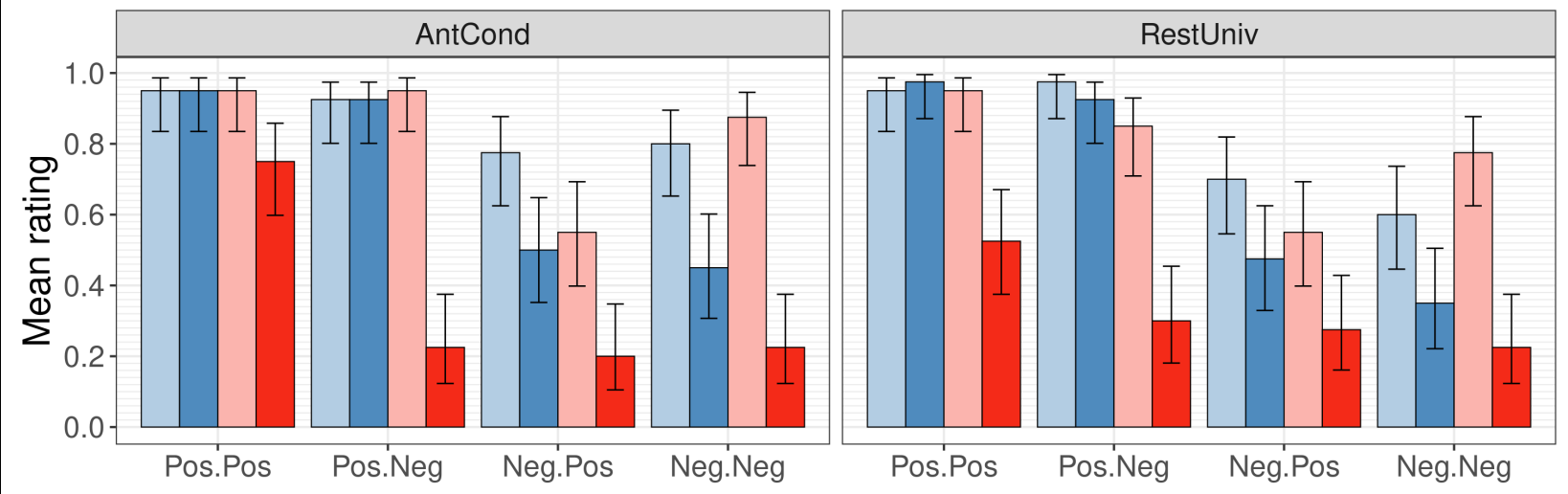

MoreThan

LessThan

AtLeast

AtMost

Figure 2: Exp. 2 (a) instructions, (b) sample trial, (c) trial summary, and (d) raw results.

Teodora Mihoc and Kathryn Davidson:

Superlative-modified numerals and negation: A multiply negotiable cost. 
(a) Exp. 2 predicted effects - general (abridged, but listing all the significant contrasts).

\begin{tabular}{rrrrr}
\hline & Est. & Std. Error & $\mathrm{z}$ value & $\operatorname{Pr}(>|\mathrm{z}|)$ \\
\hline (Intercept) & 6.07 & 1.21 & 5.028 & $<0.0001$ \\
ModMonDE & -1.87 & 1.30 & -1.436 & 0.1509 \\
ModTypeSup & -1.33 & 1.30 & -1.023 & 0.3063 \\
Pol1Neg & -4.25 & 1.19 & -3.585 & 0.0003 \\
Pol2Neg & -0.78 & 1.23 & -0.630 & 0.5288 \\
ModMonDE:ModTypeSup:Pol2Neg & -3.77 & 2.10 & -1.793 & 0.0730 \\
ModMonDE:ModTypeSup:Pol2Neg:EnvRestUniv & 6.19 & 3.02 & 2.050 & 0.0404 \\
ModMonDE:ModTypeSup:Pol1Neg:Pol2Neg:EnvRestUniv & -6.43 & 3.48 & -1.849 & 0.0644 \\
\hline
\end{tabular}

(b) Exp. 2 predicted effects - between Modifier Types (separated by Modifier Monotonicity).

\begin{tabular}{lllcrcrr}
\hline Env & Pol1 & Pol2 & Mod & OR & CI & z & p \\
\hline AntCond & Pos & Pos & MoreThan-AtLeast & 3.78 & {$[0.20,69.85]$} & 1.023 & 0.5451 \\
AntCond & Pos & Pos & LessThan-AtMost & 13.15 & {$[1.45,119.61]$} & 2.616 & 0.0089 \\
AntCond & Pos & Neg & MoreThan-AtLeast & 1.76 & {$[0.12,26.56]$} & 0.467 & 0.6402 \\
AntCond & Pos & Neg & LessThan-AtMost & 264.82 & {$[29.54,2374.44]$} & 5.701 & $<0.0001$ \\
AntCond & Neg & Pos & MoreThan-AtLeast & 4.80 & {$[1.18,19.54]$} & 2.506 & 0.0244 \\
AntCond & Neg & Pos & LessThan-AtMost & 10.71 & {$[2.28,50.38]$} & 3.434 & 0.0012 \\
AntCond & Neg & Neg & MoreThan-AtLeast & 0.67 & {$[0.12,3.64]$} & -0.532 & 0.5946 \\
AntCond & Neg & Neg & LessThan-AtMost & 6.90 & {$[1.48,32.22]$} & 2.809 & 0.0099 \\
RestUniv & Pos & Pos & MoreThan-AtLeast & 4.00 & {$[0.24,68.07]$} & 1.097 & 0.5451 \\
RestUniv & Pos & Pos & LessThan-AtMost & 96.56 & {$[6.66,1400.93]$} & 3.830 & 0.0003 \\
RestUniv & Pos & Neg & MoreThan-AtLeast & 48.06 & {$[2.34,988.59]$} & 2.870 & 0.0082 \\
RestUniv & Pos & Neg & LessThan-AtMost & 102.38 & {$[13.90,753.84]$} & 5.196 & $<0.0001$ \\
RestUniv & Neg & Pos & MoreThan-AtLeast & 2.91 & {$[0.75,11.26]$} & 1.769 & 0.0769 \\
RestUniv & Neg & Pos & LessThan-AtMost & 4.74 & {$[1.09,20.65]$} & 2.370 & 0.0178 \\
RestUniv & Neg & Neg & MoreThan-AtLeast & 0.39 & {$[0.09,1.62]$} & -1.480 & 0.2776 \\
RestUniv & Neg & Neg & LessThan-AtMost & 3.75 & {$[0.79,17.86]$} & 1.895 & 0.0581 \\
\hline
\end{tabular}

(c) Exp. 2 predicted effects - within Modifier Types (separated by Modifier Monotonicity).

\begin{tabular}{lllcrcrr}
\hline Env & Pol1 & Pol2 & Mod & OR & CI & $\mathrm{z}$ & $\mathrm{p}$ \\
\hline AntCond & Pos & Pos-Neg & MoreThan & 2.17 & {$[0.14,34.51]$} & 0.630 & 1.0000 \\
AntCond & Pos & Pos-Neg & LessThan & 1.65 & {$[0.15,17.65]$} & 0.471 & 0.6374 \\
AntCond & Pos & Pos-Neg & AtLeast & 1.01 & {$[0.07,14.96]$} & 0.010 & 0.9920 \\
AntCond & Pos & Pos-Neg & AtMost & 33.16 & {$[6.61,166.30]$} & 4.867 & $<0.0001$ \\
AntCond & Neg & Pos-Neg & MoreThan & 0.82 & {$[0.19,3.46]$} & -0.310 & 0.7568 \\
AntCond & Neg & Pos-Neg & LessThan & 1.35 & {$[0.41,4.48]$} & 0.557 & 0.5775 \\
AntCond & Neg & Pos-Neg & AtLeast & 0.11 & {$[0.03,0.50]$} & -3.280 & 0.0021 \\
AntCond & Neg & Pos-Neg & AtMost & 0.87 & {$[0.17,4.33]$} & -0.197 & 0.8614 \\
RestUniv & Pos & Pos-Neg & MoreThan & 0.42 & {$[0.02,11.43]$} & -0.590 & 1.0000 \\
RestUniv & Pos & Pos-Neg & LessThan & 3.53 & {$[0.21,58.42]$} & 1.008 & 0.6271 \\
RestUniv & Pos & Pos-Neg & AtLeast & 5.02 & {$[0.54,46.87]$} & 1.620 & 0.2103 \\
RestUniv & Pos & Pos-Neg & AtMost & 3.74 & {$[1.06,13.22]$} & 2.345 & 0.0190 \\
RestUniv & Neg & Pos-Neg & MoreThan & 1.91 & {$[0.52,7.04]$} & 1.114 & 0.5307 \\
RestUniv & Neg & Pos-Neg & LessThan & 2.19 & {$[0.62,7.72]$} & 1.399 & 0.3235 \\
RestUniv & Neg & Pos-Neg & AtLeast & 0.26 & {$[0.07,0.93]$} & -2.360 & 0.0183 \\
RestUniv & Neg & Pos-Neg & AtMost & 1.73 & {$[0.36,8.28]$} & 0.788 & 0.8614 \\
\hline
\end{tabular}

Table 2: Exp. 2 model results. $y \sim$ ModMon * ModType * Pol1 * Pol2 * Env + (1+ (ModMon + ModType + Pol1 + Pol2 + Env) $\mid$ Participant). (Statistically significant results in gray.)

Teodora Mihoc and Kathryn Davidson:

Superlative-modified numerals and negation: A multiply negotiable cost. 


\section{Experiment 3.}

4.1. GoAL. To check what happens to SMNs under [DE-ENVIRONMENT]-POS/NEG if we vary the DE environment from ANTCOND to the scope of a negated factive, as a way to test the basic insight behind V3 that the key to SMNs in ANTCOND/RESTUNIV lies with questions of monotonicity. If V3 is correct, we expect this manipulation to make no difference. In particular, an SMN under POS/NEG should be the same whether further embedded in ANTCOND or a negated factive, as both ANTCOND and a negated factive define a DE environment with an UE presupposition.

4.2. Methods. Task: Similar to Exp. 1, adapted to support minimal manipulations of the matrix-level DE environment. See Figure 3a. Sample trial: See Figure 3b. Trial summary: 16 trials, obtained by crossing the following factors: Env = matrix embedding environment (ANTCOND $=$ antecedent of conditional that here also contains a factive, MATRIXNEG = scope of matrix negation which here also contains a factive); Pol = polarity of the embedded clause (Pos = positive, $\mathrm{NEG}=$ negative $)$; and ModType $\mathrm{x}$ ModMon $=\operatorname{Mod}($ as before). See Figure 3c. Participants: 45, none excluded.

4.3. Results. For the raw means see Figure $3 \mathrm{~d}$ and for model results see Tables $3 \mathrm{a}-3 \mathrm{c}$. General effects: For all environment types, and for both CMNs and SMNs, ratings were significantly lower for ModMon=DE and POL=NEG. See Table 3a. Contrasts between Modifier Types: For all environment types, for the same level of monotonicity, SMNs (a) in POL=POS are similar to $\mathrm{CMNs}$ but in POL=NEG are sometimes worse, this effect being clearly detected for at least, especially in MAtrixNeg. See Table 3b. Contrasts within Modifier Types: Ratings for all the modifiers degrade somewhat between ANTCOND and MATRIXNEG, but for SMNs this effect was sometimes found significant, being detected in POL $=$ POS for at most and in POL $=$ NEG for both at least and at most. See Table 3c.

4.4. Discussion. Ratings for SMNs in POS/NEG had similar patterns regardless of the choice of a matrix DE environment. This is completely surprising on V1 or V2 but precisely as expected on V3. The biggest point of difference comes from AnTCOND-POS and MATRIXNEG-Pos. On $\mathrm{V} 1$ and V2, on which these conditions are merely more complex variants of ANTCOND-POS and DECL-NEG in Exp. 1, SMNs were expected to be fine in ANTCOND-POs but very degraded in MATRIX-NEG, contrary to what was observed. However, on V3, on which the presence of the factive does not just make these conditions more complex than their Exp. 1 counterparts but in fact fundamentally distinguishes MATRIXNEG-POs from DECL-NEG, endowing it with an UE presupposition, SMNs are expected to be fine in both ANTCOND-POS and MATRIXNEG-Pos, just as observed. This validates the insight from V3 that SMNs in ANTCOND(/RESTUNIV) care about monotonicity. However, although similar across the two matrix DE environments, SMNs still degraded slightly from ANTCOND to MATRIXNEG. This suggests that, while SMNs in AnTCOND(/RESTUNIV)-Pos/NEG are indeed sensitive to a deeper notion of monotonicity, as expected on V3, the specific identity of the DE operator (and possibly also whether it is actual negation) also makes a (small) difference. (Note: This brings to mind the finding from Exps. 1-2 that, although similar to ANTCOND, RESTUNIV was always slightly worse.) In addition to this, we also found a general penalty for the modifier monotonicity being DE and for the embedded polarity being negative, suggesting yet again that V1 always plays a general role also.

Teodora Mihoc and Kathryn Davidson:

Superlative-modified numerals and negation: A multiply negotiable cost. 
(a) Exp. 3 task.

In this survey you will consider a commentator for a televised card-playing game, and answer questions about how understandable the commentator is. At the beginning of the game each player gets dealt seven cards, two of which are hidden. Then in each round some rule is issued, and players can choose whether or not to bet on their own hand. A commentator, who knows what the hidden cards are for each player, discusses the player's move. You will see a player's hand and the commentator's comment, then you will be asked if you think the viewers will understand what the commentator said.

Note: In the hands that you will see, cards with a white background such as $\checkmark$ represent cards that are visible to the player, while cards with a grey background such as $\downarrow$ represent hidden cards, that is, cards that are not visible to the player but visible to the commentator.

(b) Exp. 3 sample trial. Answer options: Yes/No. The epistemic state was as illustrated across all trials.

Scyther's hand:

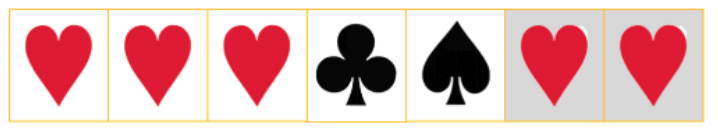

The commentator says: Scyther doesn't know that he doesn't have at most three hearts.

Do you think the viewers will understand what the commentator said?

(c) Exp. 3 trial summary. All participants saw all trials, in random order.

\begin{tabular}{lll}
\hline Env & Pol & ModType (COMP, SUP) x ModMon (UE, DE) = Mod \\
\hline \multirow{2}{*}{ MATRIXNEG } & Pos & [name] doesn't know that s/he has Mod 3 [suit] \\
\cline { 2 - 3 } & NEG & [name] doesn't know that s/he doesn't have Mod 3 [suit] \\
\hline \multirow{2}{*}{ ANTCOND } & POS & If [name] knew that s/he has Mod 3 [suit], s/he would bet differently \\
\cline { 2 - 3 } & NEG & If [name] knew that s/he doesn't have Mod 3 [suit], s/he would bet differently \\
\hline
\end{tabular}

(d) Exp. 3 raw means and their associated 95\% binomial CIs. $n=45$.

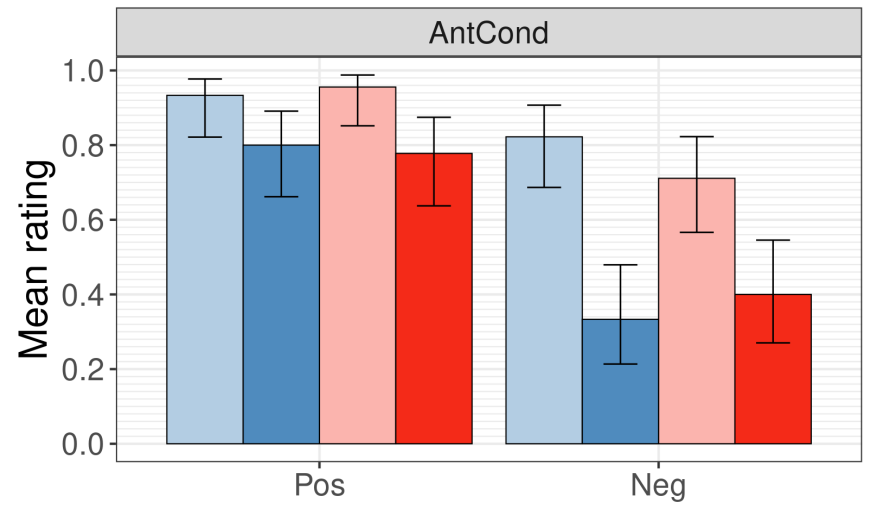

MoreThan

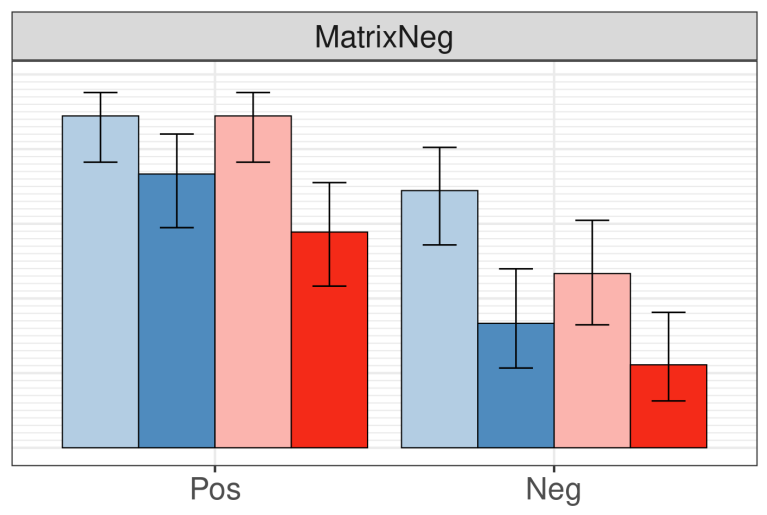

AtLeast

AtMost

Figure 3: Exp. 3 (a) instructions, (b) sample trial, (c) trial summary, and (d) raw results.

Teodora Mihoc and Kathryn Davidson:

Superlative-modified numerals and negation: A multiply negotiable cost. 
(a) Exp. 3 predicted effects - general (abridged, but listing all the significant contrasts).

\begin{tabular}{rrrrr}
\hline & Estimate & Std. Error & $\mathrm{z}$ value & $\operatorname{Pr}(>|\mathrm{z}|)$ \\
\hline (Intercept) & 4.30 & 0.96 & 4.464 & $<0.0001$ \\
ModMonDE & -2.34 & 0.94 & -2.480 & 0.0131 \\
ModTypeSup & -0.26 & 1.12 & -0.233 & 0.8161 \\
PolNeg & -2.15 & 0.99 & -2.186 & 0.0288 \\
EnvMatrixNeg & -0.52 & 0.96 & -0.539 & 0.5898 \\
ModMonDE:ModTypeSup & -0.03 & 1.22 & -0.021 & 0.9834 \\
ModMonDE:PolNeg & -0.91 & 1.10 & -0.828 & 0.4078 \\
ModTypeSup:PolNeg & -0.83 & 1.22 & -0.677 & 0.4987 \\
ModMonDE:EnvMatrixNeg & 0.07 & 1.09 & 0.067 & 0.9468 \\
ModTypeSup:EnvMatrixNeg & -0.64 & 1.30 & -0.497 & 0.6190 \\
PolNeg:EnvMatrixNeg & -0.32 & 1.11 & -0.290 & 0.7716 \\
ModMonDE:ModTypeSup:PolNeg & 1.58 & 1.47 & 1.077 & 0.2816 \\
ModPod & -0.21 & 1.51 & -0.139 & 0.8891 \\
ModMonDE:PolNeg:EnvMatrixNeg & 0.70 & 1.38 & 0.508 & 0.6112 \\
ModTypeSup:PolNeg:EnvMatrixNeg & 0.25 & 1.51 & 0.169 & 0.8661 \\
ModMonDE:ModTypeSup:PolNeg:EnvMatrixNeg & -0.61 & 1.89 & -0.321 & 0.7479 \\
\hline
\end{tabular}

(b) Exp. 3 predicted effects - between Modifier Types (separated by Modifier Monotonicity).

\begin{tabular}{llcrcrr}
\hline Env & Pol & Mod & OR & CI & z & p \\
\hline AntCond & Pos & MoreThan-AtLeast & 1.30 & {$[0.10,16.06]$} & 0.233 & 0.8161 \\
AntCond & Pos & LessThan-AtMost & 1.33 & {$[0.30,5.96]$} & 0.428 & 0.6687 \\
AntCond & Neg & MoreThan-AtLeast & 2.96 & {$[0.69,12.71]$} & 1.672 & 0.0946 \\
AntCond & Neg & LessThan-AtMost & 0.62 & {$[0.16,2.37]$} & -0.793 & 0.5065 \\
MatrixNeg & Pos & MoreThan-AtLeast & 2.47 & {$[0.34,17.98]$} & 1.023 & 0.6131 \\
MatrixNeg & Pos & LessThan-AtMost & 3.13 & {$[0.84,11.63]$} & 1.946 & 0.1033 \\
MatrixNeg & Neg & MoreThan-AtLeast & 4.38 & {$[1.17,16.40]$} & 2.505 & 0.0245 \\
MatrixNeg & Neg & LessThan-AtMost & 2.09 & {$[0.49,8.85]$} & 1.143 & 0.5065 \\
\hline
\end{tabular}

(c) Exp. 3 predicted effects - within Modifier Types (separated by Modifier Monotonicity).

\begin{tabular}{llcrcrr}
\hline Env & Pol & Mod & OR & CI & $\mathrm{z}$ & $\mathrm{p}$ \\
\hline AntCond-MatrixNeg & Pos & MoreThan & 1.68 & {$[0.20,14.37]$} & 0.539 & 0.5898 \\
AntCond-MatrixNeg & Pos & LessThan & 1.56 & {$[0.38,6.38]$} & 0.706 & 0.9598 \\
AntCond-MatrixNeg & Pos & AtLeast & 3.19 & {$[0.35,29.04]$} & 1.178 & 0.2386 \\
AntCond-MatrixNeg & Pos & AtMost & 3.66 & {$[1.02,13.11]$} & 2.282 & 0.0450 \\
AntCond-MatrixNeg & Neg & MoreThan & 2.32 & {$[0.56,9.64]$} & 1.320 & 0.3739 \\
AntCond-MatrixNeg & Neg & LessThan & 1.07 & {$[0.29,3.89]$} & 0.111 & 0.9598 \\
AntCond-MatrixNeg & Neg & AtLeast & 3.42 & {$[1.10,10.66]$} & 2.426 & 0.0305 \\
AntCond-MatrixNeg & Neg & AtMost & 3.57 & {$[0.90,14.23]$} & 2.065 & 0.0450 \\
\hline
\end{tabular}

Table 3: Exp. 3 model results. $y \sim$ ModMon * ModType * Pol * Env + $(1+($ ModMon + ModType + Pol + Env) $\mid$ Participant). (Statistically significant results in gray.)

Teodora Mihoc and Kathryn Davidson:

Superlative-modified numerals and negation: A multiply negotiable cost. 
5. Conclusion. In contrast to $\mathrm{CMNs}$, SMNs are reported to degrade under negation, though not in all DE environments. The literature offers four views: This contrast (V0) does not exist, or it exists and it is about (V1) processing cost, (V2) monotonicity and evaluativity (Cohen \& Krifka 2014), or (V3) monotonicity alone (Spector 2015, Mihoc 2020). In this paper we found the contrast does exist, pace $\mathrm{V} 0$, but is richer than processing cost, pace $\mathrm{V} 1$, the interaction between SMNs under negation being multiply negotiable, as on V2-3. For the reasons discussed above and others, ${ }^{1}$ we believe the basic interaction of SMNs with DE environments is best captured by V3 (Spector 2015, Mihoc 2020). At the same time, we conclude that V3 must be enriched to capture the evaluativity effect that inspired V2 (for an attempt see Mihoc to appear) and the general fixed costs of various DE operators that inspired V1.

\section{References}

Alexandropoulou, Stavroula. 2018. On the pragmatics of numeral modifiers: The availability and time course of variation, ignorance and indifference inferences. Utrecht: LOT.

Clark, Herbert H. \& William G. Chase. 1972. On the process of comparing sentences against pictures. Cognitive psychology 3(3). 472-517. 10.1016/0010-0285(72)90019-9.

Cohen, Ariel \& Manfred Krifka. 2014. Superlative quantifiers and meta-speech acts. Linguistics and Philosophy 37(1). 41-90. 10.1007/s10988-014-9144-x.

Cremers, Alexandre \& Emmanuel Chemla. 2017. Experiments on the acceptability and possible readings of questions embedded under emotive-factives. Natural Language Semantics 25(3). 223-261. 10.1007/s11050-017-9135-x.

Geurts, Bart \& Rick Nouwen. 2007. At least et al.: The semantics of scalar modifiers. Language 533-559. 10.1353/lan.2007.0115.

Mayr, Clemens. 2013. Implicatures of modified numerals. In Ivano Caponigro \& Carlo Cecchetto (eds.), From grammar to meaning: The spontaneous logicality of language, 139-171. Cambridge: CUP. 10.1017/CBO9781139519328.

Mihoc, Teodora. 2020. Ignorance and anti-negativity in the grammar: Or/some and modified numerals. In The Annual Meeting of the North East Linguistic Society (NELS) 50, 197-210.

Mihoc, Teodora. to appear. Modified numerals and polarity sensitivity: Between O(nly) $)_{\mathrm{DA}}$ and $\mathrm{E}(\mathrm{ven})_{\mathrm{SA}}$. In Sinn und Bedeutung $(\mathrm{SuB}) 25$, TBA.

Nouwen, Rick, Stavroula Alexandropoulou \& Yaron McNabb. 2019. Experimental work on the semantics and pragmatics of modified numerals. In Chris Cummins \& Napoleon Katsos (eds.), Handbook of experimental semantics and pragmatics, Oxford: OUP. 10.1093/oxfordhb/9780198791768.013.15.

Spector, Benjamin. 2015. Why are class B modifiers global PPIs? Handout for talk at Workshop on Negation and Polarity, February 8-10, 2015, The Hebrew University of Jerusalem.

Wason, Peter C. 1961. Response to affirmative and negative binary statements. British Journal of Psychology 52(2). 133-142. 10.1111/j.2044-8295.1961.tb00775.x.

\footnotetext{
${ }^{1}$ Further reasons include: SMNs are degraded not just under negation but under many other DE operators (e.g., without), suggesting V1 is too limited; SMNs are bad under negation even with a stereotypically positive property (e.g., Jo didn't solve \# at least 3 problems), suggesting the V2 solution for negation is incomplete, and all the DE environments where SMNs are fine seem to contain an UE presupposition (e.g., the scope of only), suggesting the V2 solution for conditionals / universals is missing a generalization.
}

Teodora Mihoc and Kathryn Davidson:

Superlative-modified numerals and negation: A multiply negotiable cost. 